\title{
Relato autobiográfico e interpretación: una concepción narrativa de la identidad personal
}

\section{Autobiographical story and interpretation: a narrative conception of personal identity}

\author{
Dante G. Duero \\ Universitat Universidad Nacional de Córdoba \\ dduero@ffyh.unc.edu.ar
}

Resumen

En este trabajo analizo el lugar de la explicación y la interpretación dentro de las ciencias en general y de las ciencias sociales e históricas en particular. Expongo los argumentos de algunos autores en favor de la
especificidad y la legitimidad del modelo narrativo dentro de las ciencias sociales e históricas. A continuación analizo la tesis que propone pensar a la identidad personal como parte de un relato que presenta grados diferentes de coherencia e integración. A partir de propuestas teóricas de diferentes autores, hago una caracterización de esta clase de narraciones y los elementos que la componen. Finalmente, reviso la tesis que afirma que la psicoterapia es un espacio para la reconstrucción y reelaboración de los relatos de los pacientes, con vistas a lograr narraciones más coherentes.

Palabras clave: Explicación; Interpretación; Identidad personal; Teoría narrativa

\section{Abstract}

In this article I analyse the role of interpretation and explanation in the field of sciences in general and in social and historical sciences in particular. I argue for the specificity of the narrative model in social and historical sciences. Then, I examine the thesis that personal identity or self is a type of autobiographic story that can show different rank of coherence and integration. Taking the proposal of different authors, I describe and I do a brief characterization of this of narrative creation and the most important element that compose its. Finally, I analyse the thesis that propose to think the psychotherapy as a place for reconstruction of the narrative production of patients for to get more coherent stories.

Keywords: Explanation; Interpretation; Personal identity; Narrative theory

\section{La interpretación y la explicación en ciencia}

Lo que preocupó a la filosofía alemana de las últimas décadas del siglo diecinueve ha sido el problema general de la historicidad de la existencia humana y su conocimiento. Para Hans Gadamer, los griegos, "primeros artífices de la concepción de mundo occidental", no tuvieron en cuenta el cambio incesante de las cosas humanas junto al ascenso y la decadencia de los pueblos y las culturas. Por el contrario, creyeron entrever una especie de orden cósmico que se mantiene pese al aparente cambio. Los griegos no admitían por tanto, la irreversibilidad como rasgo propio del ser 
humano. "Solo con el desplomo de este sustrato metafísico el problema de la historia se convierte en tema determinante para la conciencia existencial humana" (Gadamer, 1960: 34).

La ciencia griega, representada fundamentalmente por la matemática, versa sobre lo que, en tanto inmutable, puede ser conocido de una vez y para siempre. En la tradición moderna, este modelo fue desplazado hacia un interés por el mundo empírico junto con una nueva concepción acerca del conocimiento y los métodos de la ciencia, pero que se siguió enfocando al descubrimiento de leyes universales inmutables. Tal concepción estableció un vórtice sobre el cual se sostuvo prácticamente toda la filosofía del conocimiento dentro de la cultura occidental (Gadamer, 1960)

El positivismo, defensor de esta concepción ahistórica de mundo, adhirió a una tesis absolutista del conocimiento que propuso la validez universal de las verdades de las ciencias (Comte, 1844). Esta filosofía, cuya influencia, es legítimo decirlo, tiene alcance hasta la actualidad, se caracterizó por aceptar la observación y la verificación como los únicos métodos cognoscitivos válidos y por apurarse a legitimar al experimento como el camino incondicional de todo conocimiento.

Sus herederos, los empiristas lógicos, abogaron por su parte por la reconstrucción racional del conocimiento científico y el análisis sintáctico y semántico de las afirmaciones de la ciencia. Su pretensión, que era hallar un lenguaje claro y unívoco que permitiese describir y explicar los fenómenos del mundo empírico, se sustentaba en la idea del conocimiento como "representación correcta" junto con un punto de vista designativo o referencialista del lenguaje y el supuesto de una realidad del todo independiente al investigador y sus prejuicios. Tal actitud derivó en una constante preocupación por hallar la referencia empírica de los términos que conforman las teorías con sus hipótesis (Schwandt, 2000).

Para el positivismo y el neopositivismo no existiría o al menos no debiera existir, por lo demás, una diferencia de metodologías entre las llamadas ciencias naturales y las ciencias del espíritu. Cualquier disciplina que aspirase al rótulo de científica, debía adherir al modelo de "cobertura legal" (Hempel, 1966, 1967; Hempel y Oppenheim, 1948) y recurrir a la observación y el experimento como criterios últimos de validación o corroboración de sus teorías.

En el modelo de "cobertura legal", (cuya idea esencial es que las explicaciones consisten en argumentos en los que el explanandum se deduce del explanans) explicar consiste en subsumir un problema en el marco propuesto por un conjunto de premisas entre las que se halla una ley general. La explicación de un problema puede entonces inferirse de dicha ley y de las premisas que conforman las condiciones antecedentes del fenómeno de interés (Hempel y Oppenheim, 1948; Hempel, 1966 1967)

Dentro de esta filosofía no existiría diferencias entre explicación y predicción. En tanto y en cuanto una buena explicación nos proporciona información causal sobre la ocurrencia del fenómeno conduce necesariamente a predicciones adecuadas (Díez y Moulines, 1997). Por último, desde esta posición se ha sostenido que la dificultad de hallar buenas explicaciones y predicciones en el plano de las ciencias sociales e históricas sería, antes que la consecuencia de alguna particularidad en sus objetos de estudios, resultado de que sus leyes resultan, debido a la falta de precisión, oscuras y complejas o bien banales (Hempel, 1977; Ferraris, 1988). 


\section{Críticas al modelo de ciencia positiva}

Las tesis anteriores han sido cuestionadas tanto metodológica como filosóficamente. Metodológicamente, pues al presente numerosos análisis critican la validez del modelo hempeliano y sus presupuestos acerca de la explicación, inclusive dentro de las ciencias naturales (Dry, 1957; Salmon, 1990; Salmon et. Al., 1992). Y filosóficamente, debido a que parte de una serie de presupuestos ontológicos y epistemológicos que son, por lo menos, discutibles.

Junto con la antigua idea griega de que hay una esencia a descubrir y que el universo está formado por cosas muy simples (clara y distintamente cognoscibles) al ideal positivista de ciencia subyace la suposición de que el conocimiento es una representación de la naturaleza; es decir: acepta que cada representación se refiere a algún objeto (su referencia) y que la verdad de cada afirmación ha de juzgarse por el nivel de correspondencia que éstas muestra respecto al mundo (Rorty, 1979). Para el positivismo, las afirmaciones relativas a nuestras sensaciones, sentimientos, percepciones, emociones, pensamientos o recuerdos, debían hallarse adecuadamente demostradas. La falta de criterios "objetivos" para determinar con cierta certeza la presencia o ausencia de tales fenómenos, conllevaba necesariamente a descartarlos. Al no poder acceder a un modo de contrastación directa sobre los eventos que postula, a lo más, lo único que una ciencia como la psicología puede hacer es ocuparse de las correlaciones empíricas entre los actos de conducta y el medio.

Por debajo de estas afirmaciones encontramos además la pretensión de hallar un "tribunal de la razón" que dirimiese en los casos de saberes enfrentados junto con la necesidad de encontrar criterios concluyentes para producir y legitimar conocimiento. El positivismo pretendió cumplir aquí el lugar de juez capaz de evaluar los informes científicos apelando a alguna forma de representación privilegiada. Cito a Richard Rorty (1979: 288): "La idea dominante de la epistemología es que para ser racional, para ser plenamente humano, para hacer lo que debemos hacer, hemos de llegar a un acuerdo con otros seres humanos (...) La suposición de que se puede construir una epistemología es la suposición de que ese terreno [común] existe".

Por el contrario, la sospecha de que esa posibilidad de acuerdo pudiera no existir parece poner en peligro la racionalidad. Pero de hecho, ya hace mucho Friedrich Nietzche (1873) cuestionó que el hombre sea capaz de ponerse en contacto con alguna forma de conocimiento "puro" y criticó la concepción referencialista y designativa de nuestro lenguaje así como las nociones correspondencialistas sobre verdad y significado. Para este filósofo las palabras de una lengua funcionan más al modo en que las usa un poeta, cuando aplica una metáfora, que como una representación o copia de las cosas tal cual son.

Para Nietzche el investigador y el filósofo jamás aprehenden nada que tenga que ver con la "verdadera esencia" de las cosas. Ya desde su construcción, cada palabra sirve en tanto y en cuanto no describe una experiencia única y singular sino una generalidad. Es precisamente la "omisión de lo individual" lo que nos proporciona cada concepto. Remarcamos las semejanzas entre esto y aquello y, de este modo, hacemos de lo disímil algo común; llevamos a cabo "equiparación de casos no iguales" como una forma de ordenar nuestras experiencias para hacerlas inteligibles. Por ello la "verdad" es apenas: "Una hueste en movimiento de metáforas, metonimias, antropomorfismos (...) una suma de relaciones humanas que han sido realzadas, extrapoladas y adornadas poética y retóricamente y que, después de un prolongado uso, un pueblo considera firmes, canónicas y vinculantes" (Nietzche, 1873: 25). Creemos en ese enorme entramado de palabras; pero el mismo no hace sino poner las reglas con las que hemos de jugar. Olvidamos que construimos las metáforas en 
función de ciertas formas de estar insertos en el mundo, que usamos esas metáforas como intuiciones que en determinadas situaciones nos han resultado útiles para prácticas reales y concretas.

Como dice Rorty, lo que sucede es que no hay ninguna base neutral en que apoyar nuestras verdades. La justificación de una afirmación hecha siempre raíces en supuestos aceptados y no hay modo de salirnos de nuestras creencias previas y de nuestro lenguaje para hallar elementos para su justificación. Esto implica que debemos, como mínimo, dar por ciertas determinadas creencias acerca del mundo y de nosotros. No es posible salirnos de esa matriz para ubicarnos en un sitio neutral de discusión. "La idea de conocimiento como representación exacta se presta naturalmente a que se entienda como que ciertas clases de representaciones, ciertas expresiones, ciertos procesos son básicos, privilegiados y tienen carácter de fundamento. Las críticas a estas concepción (...) se ven confirmadas por argumentos holísticos de esta forma: no podremos aislar elementos básicos a no ser basándonos en un conocimiento previo de toda la estructura dentro de la que ocurren estos elementos. Por eso no podremos conseguir que la noción de representación exacta (elemento por elemento) sustituya a la realización con éxito de una práctica" (Rorty, 1979: 290)

Ahora bien, esa matriz se construye socialmente; las palabras tienen sentido en tanto y en cuanto sirven para nuestros intercambios comunicativos con miembros de nuestra propia comunidad. Las afirmaciones son justificadas por la sociedad más que por su "correspondencia con la realidad". Desde esta concepción una afirmación es "cierta" y una teoría "acertada" sencillamente si se muestra útil para un hacer. Y los criterios de aceptación de los "saberes" (en tanto "conjunto de prácticas socialmente aceptadas") son pragmáticos antes que epistemológicos (es decir, se justifican en tanto y en cuanto somos capaces de dialogar y de ponernos de acuerdo), no existiendo límites exactos para separar el modo en que genera conocimiento un artista, un científico o un hombre común.

Para Rorty es dable pensar la racionalidad antes que como un conjunto de criterios para decidir quien está en lo cierto, como una disposición a abstenerse de pensar que debe haber un conjunto especial de términos en que deben ponerse todas las aportaciones a la conversación y permanecer, en cambio, abierto a adquirir las formas de hablar de nuestro interlocutor, entendiendo que a la racionalidad y el acuerdo se llega mediante "la conversación rutinaria". Esto implica cuestionar el dogma de que la única forma de generar conocimiento es saber "que es lo que hay allí fuera" y comprender que solo podemos brindar diferentes descripciones de nuestras experiencias.

Así concebida, la fundamentación del conocimiento sería una tarea más parecida a una práctica conversacional que sirve para compartir nuestras interpretaciones que a un sistema metódico de corroboración de verdades.

\section{El giro interpretativista}

Hacia finales del siglo XIX, Wilhelm Dilthey y Hernich Rickert encabezaron un movimiento contrapuesto al del positivismo que derivó en la propulsión de modelo propio de conocimiento para las ciencias del espíritu: el de la hermenéutica y los métodos comprensivistas. Estos autores propusieron trazar una distinción "de contenido" entre ciencias de la naturaleza y ciencias del hombre. Ambos sostenían que estas áreas disciplinares se cimentaban sobre tipos diferentes de objetos de conocimiento y suponían formas diferentes de acceso cognoscitivo. Las primeras proceden a la observación del mundo exterior y se sirven de explicaciones causales; las segundas, en cambio, 
acuden a la experiencia vivida de los propios sujetos, utilizan categorías axiológicas o teleológicas, y dan por hecho que la comprensión de los significados inviste y transforma el objeto estudiado. Pero por sobre todo, en estas últimas los datos están imbuidos de significado y se organizan en función de valores y presupuestos que determinan nuestro posicionamiento frente a ellos. Como dice Paul Ricoeur (1978), el problema primordial que hay con el pensamiento hempeliano es que no reconoce diferencia entre un acontecimiento físico, "que simplemente sucede" y uno histórico, que adquiere su status de histórico tras haber sido interpretado e incluido en alguna forma de documento histórico.

A Dilthey le interesó no solo analizar el modo en que nuestro saber histórico cristaliza. Lo que pretendía era reflexionar sobre el ser humano en tanto se halla determinado por sus saberes respecto de su propia historia. Como dice Gadamer, la vida humana "...está referida a la configuración de unidades de significado duraderas", se autointerpreta y tiene "estructura hermenéutica" (Gadamer, 1960: 286). Es la autorreflexión la que nos descubre el significado de nuestras acciones entendidas desde dentro de una forma de acontecer histórica. Pero esa misma autorreflexión y autocomprensión modifica nuestros modos de existir.

De acuerdo con Dilthey, el conocimiento de lo humano no avanza de una cosa a la siguiente para abstraer generalidades; antes, las vivencias se configuran para extraer significado. Es esa vivencia inserta en un todo de un modo particular lo que le otorga sentido a la "totalidad vital". Y el significado está orientado a ese todo que se configura a partir de un "centro creador de sentido", de una "vivencia decisiva" que estructura la totalidad. Por ello el procedimiento a utilizar por parte del científico social no sería la "explicación objetivante" sino la comprensión, entendida como identificación del sujeto y el objeto. A diferencia de la explicación, que se modela bajo la forma de un juicio determinante y que subsume lo particular en la ley general, la comprensión se apoya en el "juicio reflexionante", que parte de lo particular y busca un universal que podría corresponderle pero que no necesariamente preexiste (Ferraris, 1988).

Dilthey toma al de la hermenéutica como paradigma metodológico de las ciencias del espíritu. Cuando interpretamos un texto- afirma- damos por sentado que el mismo es significativo. Es precisamente nuestra tarea como investigadores la de dilucidar su significado. Leemos cada frase presumiéndole un sentido que se derivaría de la totalidad del texto. Sin embargo la lectura de cada oración puede transformar, de un momento a otro, el significado de lo que sigue. Si nuestra primera interpretación parece volver inteligible un nuevo párrafo, entonces la aceptamos como provisoria. Si no, debemos replantear nuestros supuestos y con ello nuestra concepción de la totalidad del texto. "Una interpretación inicial provee el contexto para nuestra lectura de lo que sigue; lo revisamos y transformamos nuestra lectura en los subsiguientes pasajes (...) El texto nos cambia y dicha transformación a su vez cambia lo que nosotros entendemos del texto". (Rouse, 1987: 43).

Las acciones humanas, cree Dilthey, deben ser indagadas siguiendo un procedimiento hermenéutico parecido. Nos referimos a ellas como significativas o no y tratamos de determinar sus significados particulares redescribiéndolas en contextos diferentes que les dan sentidos nuevos. En el caso de las acciones, lo que uno debe determinar es cuales han sido los pensamientos, motivos, creencias y deseos que las acompañaron. Para ello sería preciso asociar las experiencias del sujeto cuya vida intentamos comprender con nuestras propias experiencias internas. Pero es precisamente en la distancia temporal y reflexiva- dice- que es posible la auténtica actitud comprensiva. En este punto es dónde Gadamer (1960) objeta que, en efecto, sea posible tal libertad de comprensión. 
Dilhey piensa que es posible que nuestra comprensión se libere de conceptos y prejuicios. Pero ¿puede el investigador abstraerse de sus propios intereses y prejuicios cuando interpreta un acontecimiento? ¿Lo hace acaso posible el distanciamiento y la abstracción?. Para Gadamer esa "falta de prejuicios" nunca deja de estar condicionada por algo; a la crítica supuestamente imparcial subyace siempre la influencia latente de los prejuicios. Sí no: ¿Cómo podríamos comprender lo que nos es por completo ajeno y extraño?

Es con los trabajos de Gadamer (1960) y Charles Taylor $(1985,1995)$, inspirados en la obra de Martin Heiddeger, que se da un vuelco en el pensar hermenéutico. Primero porque ambos rechazan la idea de que la hermenéutica constituya una técnica o un arte interpretativo cuya meta sea la fundamentación de una metodología para las ciencias del espíritu. Con sus propuestas nos trasladamos del lugar de la interpretación como conjunto de reglas para la generación del conocimiento histórico, al de la interpretación en tanto condición de posibilidad de todo conocimiento. Para estos autores la hermenéutica habla de algo intrínseco a nuestra condición humana, a la estructura básica de nuestra experiencia vital. Para cada uno de nosotros, nuestra experiencia del mundo y de nosotros mismos constituye siempre una experiencia en tanto existimos bajo un "horizonte" y una tradición, ambos aspectos constitutivos de nuestro modo de estar en el mundo. Antes que una fuente de prejuicio o distorsión de la realidad, son las condiciones que hacen posible interpretar y dar sentido a cada experiencia vital.

La explicación, entendida en un sentido general, es un tipo de estrategia cognoscitiva que aumenta nuestra inteligibilidad de ciertos hechos y nos permite adecuar a ellos nuestras acciones. El que tal inteligibilidad tenga lugar dependerá de si somos o no capaces de configurar los hechos que queremos explicar dentro de un entramado que los ordene. El esquema hemepeliano, con sus diferentes variantes ha pretendido- de forma más o menos satisfactoria- cumplir esta función dentro las ciencias naturales. Pero la interpretación-comprensión parece una clase diferente de herramienta cognoscitiva sui generis que nos sirve para otorgar inteligibilidad a otro tipo de experiencias, aquellas en que se involucran seres humanos y significados.

Cuando en las explicaciones sociales e históricas "interpretamos" un acontecimiento, es su inclusión en un relato lo que, al configurarlo "significativamente", nos permite hacerlo más inteligible. Lo que veremos a continuación es que las acciones humanas solo pueden ser comprendidas desde la clase de "marco hermenéutico" que conforman los relatos en los que por lo común son incluidas.

\section{El modelo narrativo}

Es dentro del giro interpretativista, aunque desde una tradición diferente a la alemana, que hacen sus aportes Arthur Danto y Hayden White. Danto (1965) sostuvo que el proceso interpretativo se halla presente en todo acto cognoscitivo y cuestionó que la explicación deba encajar en una formulación nomológica deductiva. Propuso en cambio "rehabilitar" epistemológicamente al modelo narrativo. Para Danto: "la narración no es un ornamento secundario respecto a la formulación de leyes generales; al contrario, se trata de la primera fase, indispensable, de la explicación, ya que podemos inscribir un acontecimiento en una ley explicativa solo en la medida en que dicho acontecimiento esté ya elaborado (traducido e interpretado) narrativamente " (Ferraris, 1988: 313).

Danto afirma que la naturaleza narrativa de cualquier discurso histórico deriva de que los mismos suponen siempre la vinculación de dos acontecimientos, en dónde el primero, anterior al segundo, 
cobra sentido como resultado de su lugar dentro de una trama que conduce a este último y en dónde, además, ambos acontecimientos resultan anteriores al relato del historiador. Dice Ricoeur (1978: 91): "Una consecuencia importante de la estructura de las frases narrativas consiste en que podemos cambiar la descripción que hacemos de los acontecimientos pasados en función de lo que sabemos de los futuros". Este segundo punto hace a una diferencia esencial entre explicaciones como las históricas y cualquier intento de explicación legal (aspecto que además inhabilita a aquellas para la predicción).

Por su lado, White $(1987,11)$ ha sostenido que: "la narrativa no es meramente una forma discursiva neutra que pueda o no utilizarse para representar los acontecimientos reales en su calidad de procesos de desarrollo; es más bien una forma discursiva que supone determinadas opciones ontológicas y epistemológicas con implicaciones ideológicas e incluso específicamente políticas".

Este autor cree que es necesario que la ciencia deje de considerar que nuestro conocimiento se construye proporcionando copias literales de una realidad estática y acepte los aspectos fundamentalmente dinámicos del mundo, junto con el carácter "metafórico y provisional" de los constructos que usamos para ordenar y dar sentido a nuestra experiencia. En tal sentido, White entiende que la construcción de relatos podría constituir una forma básica de asimilar nuestra experiencia a estructuras de significación que las transforman en conocimiento. La ausencia de tal estructura narrativa en nuestras explicaciones posiblemente conllevaría a experiencias ausentes de significados. Es por ello que no basta, para que haya relato (sea este histórico o literario), con que los acontecimientos se representen en un orden cronológico. Es además preciso que se revelen como dotados de una estructura, de un orden de significación que está ausente en la sola secuencia. Renegar del sentido del relato histórico para reducirlo a una serie de descripciones "empírico causales"- dice- podría llevarnos a eliminar en el proceso al fenómeno del que se quiere dar cuenta (White, 1987; véase también Veyne, 1979).

Lo particularmente interesante del asunto es que no existe, a priori, un criterio para decidir cuáles acontecimientos hemos de incluir y cuáles no dentro de un relato. Como dice Ricoeur (1978), al contar una historia los acontecimientos son importantes según contribuyan o no a hacer más inteligible la historia contada. De hecho, la misma descripción que hacemos del acontecimiento en cuestión llega a convertirse en parte de la explicación que formulamos. La relevancia del mismo depende entonces de lo que, teleológicamente hablando, llamamos el desenlace del relato. Por todo esto está en lo cierto Ricoeur cuando afirma que un acontecimiento es más que algo que sucede y que, en la experiencia humana, es lo que contribuye a la evolución de la historia que se cuenta.

En relación con este punto cabe agregar algo más y es que, dentro del relato, la trama se estructura a partir de la significación que de los hechos y situaciones vividos (como una muerte, una crisis económica, una guerra, una traición) tienen los personajes que participan en ella. Esto último significa que los acontecimientos importan en tanto resultan significativos para alguien que, inserto en esa trama, expresa intenciones, actitudes y expectativas respecto de ellos ${ }^{1}$. Es ese trasfondo psicológico

${ }^{1}$ Debemos tener presente que un relato es siempre una historia sobre algo que podría no haber pasado y sin embargo sucedió; además, el desenlace del relato implica alguna clase de consecuencia para alguien. Cada historia se constituye y evoluciona en función de las expectativas acerca de lo que es canónicamente esperable. Es una violación de tales expectativas acerca de lo posible, es el quiebre de una regularidad esperable asociada siempre a algún contenido moral relevante, a algún 
que se configura alrededor de los personajes y sus acciones lo que hace a la organización del relato y lo que posibilita una síntesis más o menos coherente de lo heterogéneo. Por ello nociones como "objetivo", "deseo", "intención", "razón, "motivo" o "proyecto" antes que elementos ideológicos prescindibles, constituyen elementos esenciales de todo buen relato. Si sustrajésemos a las acciones humanas de esta clase de contexto les quitaríamos toda inteligibilidad y sentido ${ }^{2}$.

\section{La identidad narrativa}

En Occidente compartimos la idea de que "tener un yo" o "ser sí mismo", resulta un factor clave para nuestro desenvolvimiento como seres humanos adultos (Harré, 1984). Históricamente se ha recalcado, sin embargo, la enorme dificultad para definir y caracterizar lo que entendemos "yo", siendo uno de sus rasgos constitutivos, su constante evasividad.

Para Imannuel Kant, contar con un yo significa esencialmente ser un agente responsable, consciente de la identidad numérica de la propia persona a lo largo del tiempo. La idea de ser un "sí mismo", que supone necesariamente la de considerarse un individuo, referiría así a una función organizativa básica que otorga unidad a la experiencia subjetiva y permite regular las propias conductas (Harré, 1984).

Hay autores que sostienen que alguna forma mínima de identidad personal se asienta sobre la experiencia de agentividad (la sensación de ser causa de una acción), la cual estaría intrínsecamente conectada con las de "subjetividad" y "voluntad" (Russell, 1996, 1997). En una interesante revisión sobre el tema, Shaun Gallagher (2000) usa la expresión "yo mínimo" para caracterizar este rudimentario sentimiento de mismidad al que define como "la conciencia de uno mismo como sujeto inmediato de experiencia, no extendido en el tiempo". Junto a la agentividad, el autor ubica el sentido de propiedad (esto es, la sensación de que es a mí a quien suceden ciertas experiencias). Esta forma de autoconciencia más bien básica le ha sido atribuida al niño pequeño y a algunos animales antropomorfos, ambos capaces de destrezas como reconocerse en el espejo o llevar a cabo algunas conductas imitativas (Gallup, 1970, 1977; Meltzoff y Moore, 1983).

Un segundo enfoque propone tratar al "yo" y al sentido de la "identidad personal" en términos narrativos. De acuerdo con esta propuesta, la capacidad para organizar en la memoria de modo narrativo y autobiográfico la experiencia, se hallaría estrechamente asociada con el sentimiento de identidad personal (Tulving, 1983, 1985); el "yo" sería producto de que desarrollamos narraciones sobre quienes somos. De este modo, junto con las experiencias de agentividad y propiedad, con el relato incorporamos al yo la vivencia de prolongación en el tiempo. Los hechos pasados y nuestros

deseo o intención por cumplir, a una carencia o algún logro frente a la adversidad, lo que nos habilita para la inclusión de un hecho en el cuerpo de la narración.

${ }^{2}$ En los últimos años se ha propuesto los términos "psicología popular o de sentido común" para aludir a estas prácticas interpretativas, comunes en nuestra vida ordinaria, por la cual damos sentido a la conducta propia y adjudicando a sus actores estados mentales con contenido como los que aquí menciono. De esta "herramienta cultural" se ha dicho mucho y desde posiciones divergentes. Lo que parece comúnmente aceptado es que se muestra útil al momento de negociar los significados de nuestros actos, en nuestras interacciones cotidianas. 
proyectos futuros son ubicados dentro de una historia, que nos permite fraguar en un todo unificado, coherente y significativo, los aspectos cambiantes de nuestras vivencias y que tiene la forma de relato en el que nosotros seríamos el eje referencial (Gallagher, 2000; Neisser y Fivush, 1994). La identidad no es, por ende, algo que descubrimos dentro nuestro, sino más bien una ficción que elaboramos; una especie de "metaevento" que ofrece coherencia y continuidad a la confusión de la experiencia (Ricoeur, 1985, 1990; Dennett,1991) y que se encuentra distribuido en cada elemento que ha contribuido a la elaboración ese relato: las anotaciones en cuadernos, los pasajes subrayados en un libro, las fuentes de información de nuestro ordenador, en nuestros amigos siempre propensos a darnos una referencia o un consejo (Bruner, 1991, 2002) o incluso en las producciones literarias que han marcado los estilos discursivos de una determinada época o período histórico (Bajtín, 1979).

Dentro de ese relato nosotros vendríamos a ocupar el lugar de "personaje principal". Ricoeur (1990) propone el concepto de identidad idem, para referirse al conjunto de características que reconocemos como más o menos permanentes, que aseguran cierto grado de "identidad referencial" al protagonista proporcionando coherencia a la trama. Junto a la identidad idem, entreve a la identidad ipse. Con esta expresión, se refiere a aquellos elementos cambiantes que permiten una progresión y desarrollo del personaje, dentro del drama. Nuestra identidad personal, que resultaría, de la compaginación de unos y otros rasgos, constituiría así un elemento central que es posibilitado por, pero que a la vez da lugar a, la construcción narrativa.

\section{Agentividad y relato}

Al igual que la mayor parte de los relatos, esta trama autobiográfica (que nos permita dar cuenta quienes somos y de cómo nos insertarnos en el mundo) parece hallarse enmarcada, en buena medida, por un trasfondo de intencionalidad, racionalidad y moralidad. Al construir relatos, sustentamos en serie de supuestos, incorporados a nuestro sentido común, sobre la naturaleza de nuestras acciones y el contexto moral en que se enmarcan. Así, hallamos nociones como las de sujeto individual conciente, agente libre y responsable, acción intencional, meta, deseo, propósito, motivación y voluntad. Es dentro de este trasfondo, funcional a la estructura del relato, que nuestras las acciones cobran sentido y que podemos adjudicarles, incluso, alguna connotación moral o ética.

La idea de agente parece central en este punto. En nuestros esquemas narrativos, diferenciamos la acción de la "conducta", así como de otros tipos de sucesos. Los relatos versan, mayormente, sobre agentes capaces de acciones. Pensamos que una acción es "un suceso ocasionado por un ser humano" (mi cuerpo, debe participar en la causación del mencionado cambio). Sin embargo, algunos elementos adicionales deben ser agregados. Por ejemplo, es necesario que esté despierto, conciente y al tanto de lo que estoy haciendo. Las acciones implican además otros componentes mentales, uno de los cuales es la intención. "Solamente las actividades ocasionadas intencionalmente pueden calificarse como acciones" (Van Dijk, 1998: 250). Junto con las intenciones están además los propósitos. La intención tiene a la acción dentro de su dominio; el propósito, en cambio, representa la finalidad de una acción. Unos y otros se encuentran imbricados dentro de una red o marco 
psicológico, conformado por motivos y razones que les aportan mayores o menores niveles de racionalidad y comprensibidad a la acción (Van Dijk, 1998) ${ }^{3}$.

Parece clara que la participación de al menos un agente, competente para actuar en función de intenciones y propósitos y capaz de cierto grado de responsabilidad, es necesaria para que se estructure algo parecido a una trama narrativa. Sin embargo, el hecho de concebirnos a nosotros mismos como agentes implica el habernos comprometido ya con cierto esquema de pensamiento narrativo. Esto es: un modo de pensamiento narrativo parece ser el otro lado de cierta concepción de nosotros como agentes intencionales y en cierto grado responsables, cuyas acciones (así como también intenciones y propósitos) pueden bien verse más o menos obstaculizadas al momento de alcanzar un desenlace satisfactorio.

\section{La construcción narrativa del sí mismo}

De acuerdo con Michel Foucault, la cultura de Occidente ha generado diversas "tecnologías" para la construcción de la subjetividad (Foucault 1990, 2001; Huijer, 1999). Pensarnos a nosotros mismos como sujetos responsables, nos condiciona a un modo de estar insertos en el mundo. Por el contrario, la ausencia de una identidad así como la falta un relato que dé cuenta de nuestras acciones desde un marco de intenciones y propósitos, hace imposible que nos reconozcamos como agentes. Si no se sabe quien se es, qué se quiere ni para qué, tampoco se sabe cómo actuar ni porqué. Así, un buen relato parece formar parte del conjunto de dispositivos potenciados por el ideal occidental de sujeto racional, a quien se supone capaz de hacerse cargo y de dar cuenta de sus acciones. Una marcada incompetencia para generar una adecuada narración de nosotros y nuestras acciones nos deja, en tanto, fuera de la "normalidad" y la "cordura".

Según Foucault, la epimeleia heatou, o "inquietud de sí", constituía para los griegos el momento del primer despertar ciudadano. La inquietud de sí surge al tratar de entender y significar la propia vida a fin de procurarle un orden. En sus seminarios de 1983, Foucault (2001) analiza con minuciosidad como se da este fenómeno dentro de la cultura occidental. La "inquietud de sí", nos dice, es la actitud interrogativa que hace posible el conocimiento y el posterior "cuidado de sí". Por "cuidado de sí", este autor entiende un conjunto de herramientas prácticas, de "equipamientos" útiles para autorregular los propios comportamientos, los cuales resultarían esenciales a la construcción de nuestra subjetividad.

En el extremo opuesto de la epimeleia heatou y el cuidado de sí se inscribe la stultitia o insensatez. Cuando aún no hemos cuidado de nosotros mismos lo que hay es stultitia. El stultus es aquel que no se ha ocupado de sí mismo; es por tanto una voluntad que no actúa libremente y que, al decir de Foucault, ni siquiera es voluntad.

El stultus no sabe exactamente qué es lo que quiere y por ende no quiere nunca una sola cosa por vez. Incluso quiere lo que a un mismo tiempo detesta; puede querer la gloria y a su vez lamentar no llevar una vida tranquila. Dice Foucault (2001: 135-136 - la itálica es mía-): "El stultus es ante todo quien está expuesto a todos los vientos, abierto al mundo externo, es decir, quien deja entrar en su

\footnotetext{
${ }^{3}$ Como ha sugerido en numerosos artículos el filósofo Donald Davison, aceptar la explicación por motivos o razones no nos obliga ni implica adherir a una ontológia no-determinista o "libertaria" acerca de la acción humana.
} 
mente todas las representaciones que ese mundo puede ofrecerle. Representaciones que acepta sin examinarlas, sin saber analizar qué representan (...) Por otra parte, y como consecuencia de ello, el stultus es quien está disperso todo el tiempo. El stultus es quien no se acuerda de nada, quién deja que su vida pase, quién no trata de llevarla a una unidad rememorando lo que merece recordarse y [quien no dirige] su atención, su voluntad, hacia una meta precisa y bien establecida".

Según vemos la stultitia conduciría a un modo irresponsable de ser y estar en el mundo, en dónde se actúa sin saber porqué o para qué, porque tampoco se sabe quien se es ni por tanto qué intenciones y propósitos se tienen. Para el mismo autor el stultus es el caso de quién vive su vida "sin memoria ni voluntad", sin alcanzar jamás esa relación de "plenitud consigo mismo" que es la realización de sí y que consiste en ser capaz de extender y contemplar la propia vida como una sola unidad.

¿Cómo es posible salir de esta ignorancia? De la lectura de Séneca, Foucault concluye que es preciso adquirir técnicas, destrezas y principios que nos ayuden a vivir "como corresponde". Las prácticas y "equipamientos" que hacen posible el cuidado de sí, se constituyen al modo de un conjunto de principios internos reguladores de la acción y de las relaciones que establecemos con el mundo y los demás. A éstos recurrimos cuando sucede un acontecimiento imprevisto o desventura que es preciso enfrentar y es gracias a ellos que nos preparamos para actuar en el futuro. Pero no solo eso. Estas prácticas, "se proponen o prescriben a los individuos para fijar su identidad, mantenerla o transformarla en función de una serie de fines, y ello gracias a relaciones de dominio de sí sobre sí mismo o de conocimiento de sí por sí mismo" (Foucault, 1981: 213 -la itálica es mía-).

En un trabajo de 1983, Foucault describe algunas de estas herramientas; se trata de un conjunto de ejercicios periódicos basados en la escritura (los hypomnémata) que tenían por función la "construcción de sí". La escritura a partir del autoexámen de las acciones y pensamientos del día, figura aquí como una manera de manifestarse uno a sí mismo y a los otros; escribir es mostrarse, hacerse ver, hacer aparecer el propio rostro ante el otro y ante uno mismo. Estos ejercicios conformaban una serie de prácticas de subjetivación que permitían a quienes la practicaban construir un relato coherente sobre su pasado y una idea más o menos clara acerca como comportarse en el porvenir. Dice Foucault (1983: 297 -la itálica es mía-): "Lo que es preciso construir en lo que uno escribe es su propia alma... debe poder formarse una identidad a través de la cual se lea toda una genealogía espiritual".

Desde los tiempos de la antigua Grecia y hasta la modernidad y mediante esta clase de ejercicios y anotaciones basadas en autorrelatos, el hombre occidental ha ido construyendo una especie de armazón que le permitió organizar sus experiencias y consolidar, partiendo de estos discursos sobre sí, una "estética de sí mismo" que se consideró ligada a la posibilidad de acciones morales y responsables.

\section{Características de los relatos}

Dada la importancia que, según estamos viendo, tendrían las narraciones autobiográficas sobre la constitución de nuestra identidad personal y nuestra forma de organizar nuestra experiencia vital, pareciera importante estudiar qué aspectos condicionan la conformación de los relatos y cómo los mismos se reestructuran a lo largo de un proceso como es el de la psicoterapia. 
Los comentarios de algunos especialistas reconocidos son bien sugerentes. Kenneth Gergen (1982; Gergen y Taylor, 1969), un pionero que intentó demostrar las relaciones existentes entre la autoestima y el autoconcepto, reveló cómo, entre otras cosas, las historias de las personas se modifican en relación con el tipo de relatos que se hacía sobre ellas. Según Gergen, los relatos que los demás hacen sobre quienes somos son un condicionante fundamental de nuestra experiencia de mismidad. La construcción de cada relato, dice, se halla influenciada por un sinnúmero de factores como nuestro rol social, nuestra particular posición en una situación, etc. Según sean las historias y, en definitiva, el reflejo que en sus narraciones los demás den sobre lo que somos será la imagen que generaremos respecto de nosotros mismos (véase además Shotter y Gergen, 1989).

Donald Spence (1984) por su parte, criticó el supuesto de que la efectividad del proceso psicoterapéutico dependa del trabajo de reconstrucción "histórica" de acontecimientos biográficos reales. Postuló en cambio que el quehacer terapéutico se basa en ayudar al paciente a "generar" narraciones autobiográficas más coherentes e integradas, que aquellas con las que éste llega a la consulta. Lo que fundamentalmente importaría en esta clase de procesos sería, más que la verdad histórica, la verdad narrativa y el nivel de consistencia del nuevo relato, es decir: si se ajusta a las circunstancias presentes y si ofrece a la persona una visión más o menos coherente sobre su historia y su futuro.

Según David Schafer (1981), durante la terapia, analista y analizado se proponen relatar una historia de manera que este último alcance a lograr alguna comprensión o inteligibilidad acerca de los orígenes, los significados y la importancia de sus dificultades. Entendido en estos términos, la relación terapéutica resultaría algo semejante a un "encuentro de narrativas" y el de la terapia sería un espacio de apertura para hallar nuevos "cuadros" o "metáforas" que permitan al paciente "historizar" los acontecimientos desde una estructura narrativa (diferente a la original) que conduzca asimismo a un desenlace novedoso.

Desde una perspectiva diferente Dan Mc Adams (1994, 1995, 2001; Mc Adams et. al., 1996, 2004) ha descrito asociaciones empíricas entre rasgos de personalidad (como el "neuroticismo" y la "apertura a la experiencia") y algunos índices narrativos como son el tono, el tema y la complejidad narrativa de los relatos autobiográficos de jóvenes y adultos.

Yendo a los elementos constitutivos que hacen a un relato bien estructurado, Kenneth Burke (1945) nos dice que las historias bien construidas cuentan con cinco elementos: un actor, una acción, una meta, un escenario y un instrumento, a los que se suma un problema que resulta de un desequilibrio entre cualquiera de los elementos previos (una acción impropia para alcanzar una meta, un actor que no encaja en un escenario o que entra en conflicto con una acción particular, etc.)

De forma parecida Jerome Bruner (2002) dice que todo relato requiere de una trama y que es la posibilidad de proseguir con el relato lo que determina cuales serán los acontecimientos que seleccionaremos para su conformación. Además de ello, el relato debe servirse de obstáculos para la consecución de un fin y debe hacernos reflexionar y darnos pistas que hagan comprensibles las acciones de los personajes. Finalmente sus personajes deben desarrollarse pero a un mismo tiempo deben conservar ciertos rasgos que los identifiquen dando continuidad al relato.

Por su parte, Charlotte Linde (1993) sostiene que una buena narración presenta al menos cuatro partes estructurales: a) un "resumen" o "prefacio", en el que se anuncia y enmarca el relato; b) algunas "cláusulas de orientación", con las cuales se establecen las características de los personajes y se especifica el tiempo, el lugar y las circunstancias en que ocurre el relato; c) las "cláusulas 
narrativas", que hacen al esqueleto mismo de la narración, y d) el "epílogo", con el que por lo común se indica el final del relato y que suele incluir alguna forma de "evaluación" o "conclusión" sobre el significado que el relato tiene para el narrador (cuál es su punto de vista o porqué nos lo cuenta). Este último punto es particularmente importante: en un relato, el hablante suele ofrecernos algún tipo de justificación sobre qué cuenta y porqué. Como sostiene Mijail Bajtín (1979), dentro de un relato, el autor lleva a cabo algún tipo de "actividad justificadora y conclusiva" respecto de lo relatado. El autor se ubica más allá de los acontecimientos o de las vivencias del héroe; desde allí nos ayuda a significar su vida, en tanto articulada a una totalidad que otorga unidad a lo heterogéneo.

En base a las anteriores consideraciones y basándose en los trabajos de White (1973, 1987), Linde (1993) propone diferenciar entre el discurso propiamente narrativo y otras formas que normalmente se asocian con éste, como pueden ser la crónica y la explicación. La crónica nos ofrece una secuencia de hechos o "cláusulas narrativas". Carece en cambio de los demás elementos estructurales a la narración. Es decir que no hay resumen, cláusulas de orientación, ni conclusión (cuando hay conclusión es respecto de algún evento individual más no de la secuencia narrativa como un todo).

La explicación consiste, por su lado, en la presentación de un conjunto de estamentos seguida por las razones por la que dichos estamentos deben ser creídos o considerados. Primero se presentan una serie de proposiciones de estado y luego una fundamentación para creer en su verosimilitud. Aunque hay elementos de la narración que interactúan tanto con la crónica como con la explicación- dice Linde- es conveniente diferenciar estos géneros.

De lo anterior se desprende que una narración en dónde se violara u omitiera alguno de estos principios y elementos, seguramente sería un relato incompleto, incoherente o incluso ininteligible. Un relato carente de obstáculos, por ejemplo, volvería imprecisas las intenciones del protagonista, dificultaría la progresión de la trama y por tanto imposibilitaría alcanzar un desenlace adecuado. Por otro lado, una narración en el que los personajes presentaran rasgos rígidos y contradictorios resultaría incongruente.

Podríamos, por otra parte, atender a los modos en que el desenlace elegido y la conclusión del relato condicionan la selección de los acontecimientos históricos que cabe incluir y los que no. Siendo que las partes del relato están condicionadas por la configuración global de la narración, podría suceder que los modos predominantes para seleccionar y describir los acontecimientos que componen la narración obstaculice la prosecución hacia ciertos desenlaces que fueran posibles bajo otras descripciones.

\section{La terapia: un espacio para el diálogo y la reelaboración de relatos}

Según Gadamer (1960), es en la conversación que negociamos y generamos nuevos significados. La "comprensión" consiste para él en un diálogo infinito que permite reconfigurar permanentemente nuestras perspectivas de mundo y que da lugar a nuevas interpretaciones que nos acercan de unos a otros significados. Ya dentro del contexto terapéutico parece ser el constante intercambio entre el paciente y el terapeuta en un diálogo abierto en donde el propio paciente aporta validez a cada nueva configuración narrativa, lo que posibilita el proceso de la "cura". La interpretación aquí tiene una 
función poyética antes que mimética (Ricoeur, 1978). La construcción de relatos novedosos se sostiene desde las nuevas interpretaciones; éstas resultan posibles como consecuencia de que reconfiguramos cada acontecimiento autobiográfico a la luz de descripciones diferentes a las originales.

Harold Goolishian y Harlene Anderson $(1988,1996)$ parecen acordar con esto cuando afirman que el intercambio terapéutico constituye un "entrelazamiento de ideas en el que los nuevos significados se desarrollan hacia la disolución del problema" (Goolishian y Anderson, 1996: 47). Estos terapeutas definen a los problemas como una objeción preocupada o alarmada ante un estado de cosas para la que somos incapaces de hallar una acción competente. Dicen que es la creación dialogal de una nueva narración y, por lo tanto, la apertura a una nueva mediación lo que hace al motor de cambio terapéutico. Los cito: "El poder transformador de la narración descansa en su capacidad para rerelatar o re-relacionar los hechos de nuestras vidas en el contexto de un significado nuevo y diferente" (Goolishian y Anderson, 1996: 47-48).

Al respecto cabe decir que es la inclusión de la experiencia del paciente en un relato con un desenlace particular lo que define el problema y nos permite dar inicio al proceso terapéutico. Un problema por lo general supone una incongruencia dentro de la trama de un relato, que conduce a un desenlace que es valorado por el paciente como poco satisfactorio. Pero la definición de ese problema depende del contexto narrativo del cual se deriva su significado. Es la propia configuración de la trama del relato la que conduce al paciente a definir al problema de un modo y no de otro. "Las narraciones permiten (o impiden) una percepción personal de libertad o competencia para dar sentido y para actuar (mediación). Se puede considerar que los problemas que se tratan en terapia emanan de narraciones sociales y autodefiniciones que no brindan una mediación que sea eficaz para las tareas implícitas en su autonarraciones ${ }^{4}$. La terapia brinda la oportunidad de desarrollar nuevas y diferentes narraciones que permitan una gama ampliada de mediación alternativa a la di-solución del problema. Y quienes visualizan la terapia como exitosa experimentan la realización de esta nueva mediación narrativa como libertad y liberación" (Goolishian y Anderson, 1996: 51).

Como terapeutas, y ante la demanda de un paciente, por lo común nos preguntamos: ¿qué puede estar provocando, dentro de este relato, el desajuste que conduce a un desenlace insatisfactorio?. Podemos examinar entonces los diversos componentes de la trama (atender a cómo se ha caracterizado al personaje, sus acciones, intenciones y propósitos; a qué tipo de desenlace conduce la historia y qué conclusión saca el paciente de ello, etc.) para luego orientarnos, junto con el paciente, hacia la "solución", la "reformulación" o bien la "disolución" del problema ${ }^{5}$. La sola

\footnotetext{
${ }^{4}$ Por ello Goolishian y Anderson (1996) recomiendan prestar atención a las autodescripciones que el paciente hace de sí, pues estas operan de forma crucial en la organización del comportamiento y la experiencia.

${ }^{5}$ Nos centramos en la "solución" cuando buscamos, por ejemplo, "acciones" más eficaces que conducen a mayores niveles de "satisfactoriedad en la intención" y/o "el propósito" del paciente (Van Dijk, 1998). Por otro lado, una estrategia como analizar las intenciones y propósitos y el marco psicológico de "razones" y "motivos" en que se sustentan, muy probablemente lleve a la "reformulación" o "disolución" del problema. Con la reconstrucción de la historia a partir de una nueva formulación del problema y de su posible solución vendrá la búsqueda de un desenlace con una conclusión diferente.
} 
relaboración del relato, como la inclusión de acontecimientos o circunstancias que han sido omitidos, puede conducir a la desaparición del problema o a su consideración desde un perfil diferente.

Sobre este aspecto parecen trabajar los terapeutas Tom Andersen (1991) y Steve de Shazer (1994) cuando proponen que el terapéutico sea un espacio para la búsqueda de acontecimientos "inusuales" y la construcción de una "historia de logros". Ambas estrategias apuntarían a focalizar los relatos sobre los aspectos funcionales de la experiencia de los pacientes. Estos remarcan la relevancia de estas herramientas como un modo de hallar la solución al problema de consulta desde un marco distinto al que estructura las narrativas típicas de los pacientes. Michael White (1997), por su parte, habla de las "excepciones" a la regla, como aquellos antecedentes atípicos a tomar en cuenta para la construcción de nuevos relatos. Las situaciones en las que el paciente se orienta de forma novedosa frente a un problema constituyen, según parece, la clase de excepciones que por lo común no se incluye en sus relatos oficiales. La labor terapéutica consiste aquí en ayudar a consolidar un contexto narrativo diferente al que el paciente trae a terapia, a fin de que permitirle configurar una nueva trama que incluya a tales excepciones.

Creo que es interesante comentar en este punto que, más de cuarenta años atrás, muy previamente a la puesta en boga de los modelos narrativos en psicología, Carl Rogers (1966) hizo detalladas descripciones sobre el la forma en que los pacientes modifican su visiones de sí mismos y sobre cómo cambiaban los modos de relatar sus experiencias personales gracias al proceso terapéutico. Entre otras cosas, cuenta que éstos lograban incluir y aceptar experiencias, sentimientos y emociones nuevas; que durante las entrevistas se describían a sí mismos como mejor integrados y capaces de tolerar ciertos niveles aceptables de contradicción; que aprendían a diferenciar sus propias experiencias subjetivas, respecto de las de los demás y respecto del mundo y por sobre todo que comenzaban a aceptar lo que hoy podríamos llamar otras narraciones o relatos sobre sí mismo y su propia historia, sin que ello menoscabase la propia imagen.

Respecto de ello (es decir: de los cambios que se producen en la imagen que los pacientes tienen de sí y de los demás), creo que puede ser aclarador citar extractos de algunas entrevistas tomadas durante un estudio piloto, que tuvo por propósito analizar los procesos implicados en la construcción de la identidad personal a través de relatos autobiográficos. El mismo, realizado en Córdoba (Argentina) durante el año 2004, se llevó a cabo sobre una muestra de 15 adultos universitarios, de entre 18 y 30 años, que habían tenido algún tipo de experiencia terapéutica. Cuando se les preguntó a los participantes "¿qué creían que había cambiado en ellos la terapia", la mayoría adujo que, gracias a ella, habían modificado su imagen de sí y habían conseguido incorporar a sus autodefiniciones, nuevos rasgos que incluían sentimientos, emociones, deseos y capacidades para actuar, hasta entonces omitidos. Asimismo, dijeron haber conseguido resignificar situaciones, acontecimientos, acciones y relaciones que consideraban importantes, desde una nueva perspectiva que les permitía un enfoque más amplio y novedoso. Un ejemplo para citar es Nadia, quien había hecho psicoanálisis por más de tres años. Esta mujer de 30 años, contó que al comienzo del proceso tenía una noción "menos acabada" de lo que era y de su historia y que gracias a ella pudo "arribar a una imagen más ordenada de ella misma" así como de las "personas que le eran significativas". A continuación trascribo parte de su respuesta: "Para mi fue un encuentro. Poder dar cuenta de mi infancia y de mi historia en un lenguaje propio. Fue como volver propio algo que no era consciente, como una imagen... hacerme cargo de la percepción que tenía de mí misma y discriminar la que los otros tenían de mí para ver que era y que no mío (...) La terapia me ayudó además a recuperar ciertas expresiones y resignificarlas proponiendo interpretaciones". 
Refiriéndose luego al relación que había mantenido con su padre, comentó cómo la terapia le posibilitó "resignificar las actitudes de él" y "comprenderlas desde un marco distinto". La cito: "Entendí que mi padre era producto de su historia, que estaba condicionado y también era víctima de sus actos. Comprender eso me permitió comenzar a acercarme a él, contarle cosas, dejar de tenerle miedo".

Otra mujer, de 27 años que había participado en varios procesos terapéuticos con enfoques teóricos distintos, habló acerca de cómo, gracias a la terapia, logró "modificar las formas en que se representaba algunos problemas" y sobre cómo consiguió arribar a una "nueva composición de elementos". Esta entrevistada describió al del diálogo terapéutico como un espacio que le permitió tomar sus problemas como una "materia con la que jugar". El terapeuta: "tomaba mi mundo de asociaciones y me ayudaba a reconfiguralo, a reconfigurar mi mundo de asociaciones y mis miradas sobre la realidad... con ello la lógica de mis asociaciones cambió".

En aparente concordancia con esto un contemporáneo nuestro, Vittorio Guidano (1991), afirma que durante el proceso terapéutico los pacientes parecen aprender a diferenciar entre los acontecimientos y la interpretación que puede hacerse de ellos, que discriminan diferentes estados subjetivos y que aprenden a negociar con los significados que otras personas otorgan a acontecimientos compartidos, aspectos todos que han de ser tenidos en cuenta durante la elaboración de un buen relato autobiográfico.

Es significativo que todos estos psicólogos, desde Rogers hasta White, parezcan coincidir en que la función del terapeuta es posibilitar un espacio abierto de dialogo, aceptación y escucha que lleve a la persona a construir descripciones enriquecedoras, positivas y coherentes acerca de sí, con vistas a alcanzar una concepción de sí mismo y su vida futura más satisfactoria y benevolente. Transcribo a continuación lo que dijo la primera entrevistada, luego de que se le preguntara cómo describía al proceso terapéutico: "La terapeuta me señalaba y confrontaban aspectos de lo que decía que resultaban contradictorios. Sin embargo no hacía hincapié en esas contradicciones. Solamente las marcaba. Y siempre preguntaba mucho. Me preguntaba que podía significar o si me parecía una interpretación que ella proponía. Yo era la que decidía. Al finalizar cada sesión hacía un cierre. Recopilaba lo hablado y me lo devolvía con un cierto orden. Luego me preguntaba si podía ser así".

Otro muchacho, de 26 años, y que al momento de ser entrevistado se encontraba realizando un tratamiento con un terapeuta cognitivo hizo el siguiente comentario: "Yo venía con un montón de problemas. El [el terapeuta] supo esperar y después preguntar... marcaba con mis propias palabras, con fragmentos míos, mis propios errores (...) Me hacía reflexionar mucho. Con esas preguntas... yo volvía sobre mis palabras. Ese análisis hizo que yo fuera revisando tales palabras (...) Todo aquello me ayudó a dejar de echar culpas a terceros y afrontar mis problemas... Cuando frené y dije: tu forma de ser te está creando un conflicto frené y cambié".

En relación con este último comentario, cabe citar una observación de Goolishian y Anderson (1973) quien cree que durante la terapia el terapeuta y el paciente trabajan fundamentalmente en la restauración del "sentido de agencia", a fin de que el segundo llegue a sentirse mejor capacitado para iniciar acciones competentes. "...La agencia e intención son funciones de las narrativas que desarrollamos: nuestras narrativas nos dan un sentido de agencia (...) El cambio en terapia no es la resolución de problemas sino el restablecimiento del sentido de agencia que es paralelo al desarrollo de nuevas narrativas $y$, en consecuencia, nuevas intenciones que sean consistentes con esa agencia. 
Si la experiencia terapéutica es vivida como exitosa, lo que la gente experimenta entonces es una sensación de libertad: ahora puede tomar la acción por sí misma" (Goolishian y Anderson, 1973: 311).

\section{Conclusión}

Luego de hacer una revisión del lugar que la explicación y la interpretación han tenido en el campo de las ciencias, he ofrecido diferentes argumentos en favor de la especificidad y la legitimidad del modelo narrativo como forma explicar en ciencias sociales e históricas. Seguidamente he analizado la tesis, que en la actualidad es defendida y consensuada desde las teorías narrativas, según la cual la identidad personal se construye por medios narrativos. Basándome en diferentes aportes, he sostenido que las características y los niveles de integración y coherencia que logramos en nuestros relatos autobiográficos, así como el modo en que nos insertamos dentro de ellos, determina nuestra vivencia de nosotros mismos y el significado que le otorgamos a nuestra experiencia. He afirmado que al menos una buena parte del trabajo psicoterapéutico consiste en ayudar al paciente a buscar interpretaciones significativas pero más provechosas, con vistas a lograr una mejor comprensión acerca de los orígenes, significados e importancia de sus dificultades. Finalmente, y siguiendo las propuestas narrativas de Goolishan, Anderson y otros, sugerí que nuestros esfuerzos terapéuticos deben tender a favorecer, durante los intercambios conversacionales entre el paciente y el terapeuta, a la reconstrucción narrativa autobiográfica así como también a una "deconstrucción de las prácticas domésticas aceptadas".

Creo importante en este punto remarcar que un proceso deconstrucción y reconfiguración como el descrito no se realiza jamás sobre el "texto" interpretado, sino sobre su autor, que en este caso es el propio narrador. Es decir que el "relato" no es algo acabado y que pueda entenderse al margen de nuestras experiencias de mundo, ni viceversa. Es preciso comprender la dimensión relizativa del discurso (Ricoeur, 1984) y a un tiempo entender que la "realidad" no es algo que se separe de nuestros relatos; que las fronteras entre lo "interior" y lo "exterior", o entre lo "lingüístico" y lo "extralingüístico" del relato es producto de los métodos que los investigadores aplican para organizar su análisis y no algo intrínseco a la naturaleza del relato. Por el contrario, ningún relato tiene lugar a un costado de las experiencias humanas: no hay ninguna experiencia extranarrativa que alcance a formar parte, hablando en un sentido fuerte, del mundo conocido por los hombres (Ricoeur, 1984).

Tal como he abordado el problema, significaría haber caído en una confusión conceptual grosera si se tiende a hacer la artificiosa y equívoca distinción entre un mundo de "relatos" o "narraciones" y un mundo "extranarrativo" completamente independiente. Relato y experiencia son ambos aspectos que hacen a cualquier proceso narrativo. Como dijera el filósofo Daniel Vera (comunicación personal) haciendo lo que creo una muy pertinente paráfrasis de Kant: "la experiencia sin relato es ciega y el relato sin experiencia, algo vacío". Llegados aquí me parece apropiado afirmar con Ricoeur (1984) que la experiencia humana posee carácter "prenarrativo", que toda ella es una actividad y una pasión en búsqueda de un relato.

\section{Referencias}

Andersen, Tom. (1991). El equipo reflexivo. Gedisa, 1994. 
Anderson, Harlene y Goolishian, Harold A. (1988). Human system as linguistics systems: Preliminary and evolving ideas about the implications for clinical theory. Family Process, 27, 371-393.

Anderson, Harold y Goolishian, Harlene. A. (1996). El experto es el cliente. En Sheila McNamee y Kenneth J. Gergen (Eds.). La terapia como construcción social (pp. 45-60). Barcelona: Paidós, 2002.

Bajtín, Mijail. (1979). Estética de la creación verbal. Madrid: Siglo XXI, 1982.

Bruner, Jerome. (1991). Actos de significado. Más allá de la revolución cognitiva. Madrid: Alianza.

Bruner, Jerome. (2002). La fábrica de historias. México: Fondo de Cultura Económica.

Burke, Kenneth. (1945). A Grammar of Motives. New York: Prentice Hall.

Comte, August. (1844). Discursy sur l'espirit positif. Vol. XI. Citado por Ferraris (1988).

Danto, Arthur. C. (1965). Analytical Philosophy of History. Cambridge: Cambridge U.P. Ed. castellana (parcial, cap. 1, 7 y 8). Historia y narración. Ensayos de filosofía analítica de a historia. Barcelona: Paidós, 1989.

De Shazer, Steve. (1994). En el principio las palabras eran magia. Barcelona: Gedisa, 1999.

Dennett, Daniel. (1991). La conciencia explicada. Barcelona: Paidós, 1995.

Díez, José A. y Moulines, Ulises C. (1997). Fundamentos de Filosofía de la Ciencia. Barcelona: Editorial Ariel, 1999.

Dray, William. (1957). Law and Explanation in History. Oxford: Oxford U.P.

Ferraris, Maurizio. (1988). Historia de la hermenéutica. Madrid: Ediciones Alkal.

Foucault, Michael. (1981). Subjetivité et vérité. Dits et Ecrits, IV. Paris : Gallimard.

Foucault, Michael. (1983). L’ecriture de soi. Corps écrit, 5, 3-23.

Foucault, Michael. (1990). Tecnologías del yo. Barcelona: Editorial Piadós-U.A.B.

Foucault, Michael. (2001). La hermenéutica del sujeto. México: Fondo de Cultura Económica, 2002.

Gadamer, Hans. G. (1960). Verdad y método. Salamanca: Editorial Sígueme, 1998.

Gallagher, Shaun (2000): "Philosophical conception of the self: implications for cognitive science". Trends in Cognitive Sciences, Vol. 4, No 1.

Gallup, Gordon G. (1970). Chimpanzees: self-recognition. Science, 167, 86-87

Gallup, Gordon G. (1977). Self recognition in primate. American Psychologist, 32, 329-338.

Gergen, Kenneth. (1982). Toward transformation in Social Knowledge. New York: Springer- Verlag.

Gergen, Kenneth y Taylor, M. G. (1969). Social Expectancy and Self Presentation in a Status Hierarchy. Journal of Experimental Social Psychology, 5, 79-92. 
Goolishian, Harold y Anderson, Harlene (1973). Narrativa y self. Algunos dilemas posmodernos de la psicoterapia. En Dora F. Schnittman (Comp.). Nuevos paradigmas, cultura y subjetividad (pp. 293-311). Barcelona: Paidós.

Guidano, Vittorio. (1991). El sí mismo en proceso. Buenos Aires-Barcelona-México: Editorial Paidós, 1994.

Harré, Rom. (1984). Personal Being. Cambridge. Massachusetts: Harvard University Press.

Hempel, Carl. (1966). Philosophy of Natural Science. New Jersey: Prentice- Hall.

Hempel, Carl. (1967). La explicación científica. Barcelona: Paidós, 1988.

Hempel, Carl y Oppenheim, Paul. (1948). Studies in the Logic of Explanation. Philosophy of Science, $15,567-579$.

Huijer, Marli. (1999). The aesthetics of existence in the work of Michael Foucault. Philosophy \& Social Criticism, 25, 2, 61-85.

Linde, Charlotte. (1993). Life Stories. The creation of coherence. Oxford: Oxford University Press.

Mc Adams, Dan P. (1994). Can personality change? Level of stability and growth in personality across the lifespan". En Todd F. Heatherton and Joel Lee Weinberger. (Eds.). Can Personality Change (pp. 299-313). Washington, DC: APA Books.

Mc Adams, Dan P. (1995). "What do we know when we know a person?. Journal of Personality, 63, 365-396

Mc Adams, Dan P. (2001). The person: An integrated introduction to personality psychology. New York: Wiley.

Mc. Adams, Dan P.; Hoffman, Barry. J.; Mansfield, Elizabeth. D. y Dray, Rodney (1996). Themes of agency and communion in significant autobiographical scenes. Journal of Personality, 64, 339-377.

Mc Adams, Dan P. ; Anynidoho, Nana A.; Brown, Chelsea, Huang, Yi T., Kaplan, Bonnie y Machado, Mary A. (2004). Traits and Stories: Link Between Dispositional And Narrative Features of Personality. Journal of Personality, 72, 4, 762-784.

Meltzoff, Andrew y Moore, Keith M. (1983). Newborn infants imitate adult facial gestures. Child Development, 54, 702-709.

Neisser, Ulric y Fivush, Robyn. (1994). The Remember Self: Contribution and Accuracy in the SelfNarrative. London: Cambridge University Press.

Nietzche, Friedrich. (1873). Sobre verdad y mentira en el sentido extramoral. Barcelona: Editorial Tecnos, 1990.

Polonoff, David. (1987). Self-deception. Social Research, 54 (1), 45-53.

Ricoeur, Paul. (1978). Historia y Narratividad. Barcelona: Paidós, 1999.

Ricoeur, Paul. (1984). Educación y política. Buenos Aires: Editorial Docencia. 
Ricoeur, Paul. (1985). Temps et Récit. París: Seuil.

Ricoeur, Paul. (1990). El sí mismo como otro. Madrid: Siglo XXI, 1996.

Rogers, Carl R. (1961). El proceso de convertirse en persona. Buenos Aires-Barcelona-México: Editorial Paidós, 2003.

Rogers, Carl R. (1966). Psicoterapia Centrada en el Cliente. Buenos Aires: Editorial Paidós.

Rorty, Richard. (1979). La filosofía y el espejo de la naturaleza. Madrid: Ediciones Cátedra, 1989.

Russell, James. (1997). Como los trastornos ejecutivos pueden dar origen a una inadecuada teoría de la mente. En James Russell (Ed.). El autismo como trastorno de la función ejecutiva (pp. 245-296). Argentina: Editorial Panamericana, 1998.

Russell , James. (1996). Agency: its role in mental development. Erlbaum (UK): Taylo \& Francis.

Salmon, Marilee. (1990). Four Decades of Scientific Explanation. Mineapolis: The University of Minnesota Press.

Salmon, Marrilee. (1992). Scientific Explanation. En Marrilee Salmon; John Earman; Clark Glymour; James, G. Lennox; Peter Machamer; J. E. McGuire; John Norton; Wesley Salmon y Kenneth Schaffner. (Eds.). Introduction to the Philosophy of Science (pp. 7-41). New Jersey: Prentices Hall, Englewood Cliffs.

Schafer, Roy. (1981). Narration in Psychoanalytic Dialogue. En: William. J. T. Mitchell (Ed). On Narrative. Chicago; University of Chicago Press.

Shotter, John y Gergen, Kenneth . J. (1989). Texts of identity. London: Sage.

Shwandt, Thomas. (2000). Three Epistemological Stance for Qualitative Inquiry. En Norman K. Denzin y Ivonna S. Lincoln. (Eds.). Handbook of Qualitative Research (pp. 189-213). London. New Delhi: Sage Publications, Inc.

Spence, Donald. (1984). Narrative Truth and Historical Truth: Meaning and Interpretation in Psychoanalysis. New York: Norton.

Taylor, Charles. (1985). Philosophical Papers. Cambridge: Cambridge University Press.

Taylor, Charles. (1979). Interpretation and the Sciences of Man. En Paul Rabinow y William Sullivan (Eds.). Interpretative Social Sciences. A reader (pp. 33-81). Berkeley: University of California Press.

Tulving, Endel. (1983). Elements of Episodic Memory. London: Oxford Clarendon Press.

Tulving, Endel. (1985). Memory and Consciousness. Canadian Psychology, 26, 1-12

Van Dijk, Teun .A. (1998). Texto y Contexto. Madrid: Editorial Cátedra.

Veyne, Paul (1979). Writing History: Essay on Epistemology. Middletown: Wesleyan University Pres, 1984. 
White, Hayden. (1973). Metahistory. The historical Imagination in Nineteenth-Century Europe. Baltimore- London: The Johns Hopkins U.P.; ed. castellana: Metahistoria. La imaginación histórica en la Europa del siglo XIX. México: FCE, 1992.

White, Hayden. (1987). El contenido de la forma. Barcelona: Ediciones Paidós, 1992.

White, Michael. (1997). El enfoque narrativo en la experiencia de los terapeutas. Barcelona: Gedisa, 2002.

\section{Historia editorial}

Recibido: 19/10/2005

Primera revisión: 07/02/2006

Aceptado: 22/03/2006

\section{Formato de citación}

Duero, Dante. G.. (2006). Relato autobiográfico e interpretación: una concepción narrativa de la identidad personal. Athenea Digital, 9, 131-151. Disponible en http://antalya.uab.es/athenea/num9/duero.pdf

Dante G. Duero. Realizó su doctorado en Psicología en la Universidad Nacional de Córdoba, sobre la formación de conceptos mentales en preescolares. Actualmente es docente de dicha universidad y realiza estudios posdoctorales sobre la temática "Narrativa e Identidad Personal", como becario del CONICET.

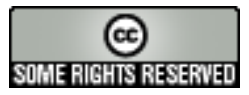

Este texto está protegido por una licencia Creative Commons.

Usted es libre de copiar, distribuir y comunicar públicamente la obra bajo las siguientes condiciones:

Reconocimiento: Debe reconocer y citar al autor original.

No comercial. No puede utilizar esta obra para fines comerciales.

Sin obras derivadas. No se puede alterar, transformar, o generar una obra derivada a partir de esta obra.

\section{$\underline{\text { Resumen de licencia }}$}

Texto completo de la licencia 